\title{
Bacterial image segmentation algorithm based on improved level set
}

$$
\text { Xian-qi Cao }{ }^{1, a} \text {, Jia-qing } \mathrm{Miao}^{2,3, \mathrm{~b}}
$$

${ }^{1}$ College of Applied Technology, University of Science and Technology LiaoNing, China

${ }^{2}$ School of Mathematical Sciences, University of Electronic Science and Technology of China, China

${ }^{3}$ The Engineering \& technical College of Chengdu University of Technology, China

acaoxq905@163.com, ㅁmjq_011114117@163.com

\begin{abstract}
Keywords: Sewage treatment, Bacteria image segmentation, Level set, CV model, LBF model.
Abstract. The ever - worsening water pollution has prompted the emergence of a large number of sewage treatment plants; meanwhile, the activated sludge process has been developed rapidly. The species, quantity and the stage of growth of the microorganisms in the sewage treatment by activated sludge process is the major determinants of sludge settling performance. So the level set and its improved methods of bacterial image segmentation on CV model and LBF model are studied in the paper, and then the bacterial image is segmented and identified in the sewage treatment process through microscopic examination of activated sludge microorganisms. The results show that LBF variational level set model for bacterial image segmentation is more efficient, stable and robust. Therefore, in sewage treatment, the sludge settling performance can be predicted according to the results of the segmentation, so as to take measures to further improve the process.
\end{abstract}

\section{Introduction}

Recently, with the improvement of people's living standard and the increasing demand for water, sewage treatment technology is rapid development, and one of the main processes is activated sludge, which is based on the organic pollutants in the sewage is constantly adsorbed and decomposed by microorganisms so as to achieve sewage purification. Number and types of microorganisms that affect the efficiency of sewage digestion directly in process play a decisive role. The main purpose of this paper is to find an optimal method to understand and control the quantity, type and growth stage of microorganisms in the sludge. With the rapid development of digital image processing technology, many applications in this field have been developed. In this paper, the improved level set method is used to design and realize the segmentation of the microorganism image in the activated sludge process. For example, in activated sludge purification system, the microbial images of sludge in each period were segmented by level set method [1], and then the processing performance of the system can be understood by the number, type and morphology of the microorganisms. In recent years, researchers have devoted a lot of research work on the segmentation of bacterial images by active contour models. It has many advantages, for example, the curves represented by the level set equation in the evolution may be naturally merged or interrupted [2], so that the topological changes of bacterial image can be processed automatically, and the level set equation is always maintained at a fixed grid, which can make the numerical scheme more efficient.

\section{C-V Model}

\section{Model Building}

First, an evolution curve $C$ is defined in $\Omega$ range, and it is $\Omega$ 's boundary of an open subset $\omega$ (in other words $\omega \subset \Omega$ and $C=\partial \omega)$. In the following content, insideC represents the area of $\omega$, and outside $(C)$ represents the area of $\Omega / \varpi$.

The method is based on the minimization of image energy partitioning with a simple example to illustrate the basic idea of the model [3]. Assuming that the image $\mu_{0}$ is composed of two piece-wise constant intensity regions of $\mu_{0}^{i}$ and $\mu_{0}^{0}$, the further assumption is that the target to be detected is 
represented by the $\mu_{0}^{i}$ region, and $C_{0}$ represents the target boundary, and then we can get the target internal area $\mu_{0}=\mu_{0}^{i}$, the external area $\mu_{0}=\mu_{0}^{\sigma}$. Consider the following fitting term:

$$
\begin{aligned}
F_{1}(C)+F_{2}(C)= & \int_{\text {inside }(C)}\left|\mu_{0}(x, y)-c_{1}\right|^{2} d x d y \\
& +\int_{\text {outside }(C)}\left|\mu_{0}(x, y)-c_{2}\right|^{2} d x d y
\end{aligned}
$$

where $C$ is any other variable curve, and the values of constant $c_{1}$ and $c_{2}$ that depend on the curve $C$ are the average values of inside $C$ and outside $C$ of $\mu_{0}$. In this simple example, it is obvious that the target boundary $C_{0}$ is the minimum of the fitting term.

$$
\inf _{c}\left\{F_{1}(C)+F_{2}(C)\right\} \approx 0 \approx F_{1}\left(C_{0}\right)+F_{2}\left(C_{0}\right)
$$

This is obvious, for example, if the curve $C$ is outside the target, then there are $F_{1}(C)>0$ and $F_{2}(C) \approx 0$, and if the curve $C$ is inside the target, then there are $F_{1}(C) \approx 0$ and $F_{2}(C)>0$, and if a curve is part of inside and the other part is outside, then there are $F_{1}(C)>0$ and $F_{2}(C)>0$. Finally, if $C=C_{0}$, then the fitting term is minimized, and the curve $C$ is exactly on the target's boundary.

We note that the key of the algorithm is not the time based on PDE in the equation $\phi$ [4], and it can be solved numerically by the finite difference method, which is the same as that of the minimization problem [5].

\section{Experimental Results}

The model is applied to the real bacteria images with different contours and shapes, at the same time, the active contour evolution and the piece-wise constant of the original image $\mu_{0}$ are given ( $c_{1}$ and $c_{2}$ average). In order to detect the internal contour of bacterial image automatically and to ensure the global minimum solution, the approximate values $H_{2, \varepsilon}$ and $\delta_{2, \varepsilon}$ of Heaviside and Dirac delta's equation (on condition of $\varepsilon=h=1$ ) are used, where only the length parameter $\mu$ has scaling function. If it needs to detect the boundaries of any size as much as possible, then $\mu$ should take a small value. And if it needs to detect large targets (such as block targets), then $\mu$ should take a larger value. The exact value of $\mu$ and the initial level set equation $\phi_{0}$ are given each time in the experiment.

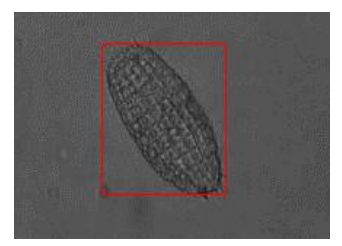

Colepidae
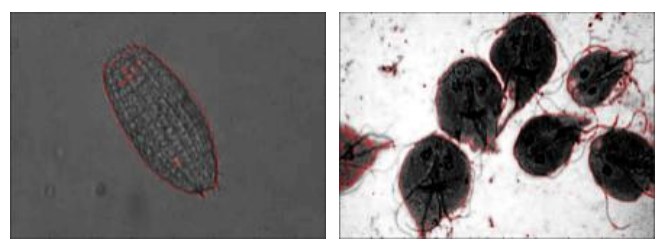

(b) Segmentation results of C-V model

Fig. 1 Various bacterial segmentation results of $\mathrm{C}-\mathrm{V}$ model.

The contour and shape of the $\mathrm{C}-\mathrm{V}$ model are detected automatically, and the second initial curves are not considered (see Fig. 1). Because the level set is adopted in the model, it can change the topology of the image automatically. The model also shows that the model can detect different targets with different intensities and different boundaries. The inner contour of the same image can be detected automatically, which is due to the evolution speed that has global dependence, and the curve is closer to the target. Through this model, the target of the boundary defined by the image gradient information or the target with very smooth boundary can be detected, which cannot be detected by the traditional active contour model. 
But the model still has many limitations, such as image segmentation effect is not ideal for high noise, and there are more false segmentation results, and even some bacteria image segmentation is imperfect, which has a lack of segmentation.

\section{LBF Variational Level Set Model}

\section{Model Building without Re Initialization}

A variational formula of the geometric active contour makes the evolution of the level set equation close to a signed distance function, which can eliminate time-consuming re initialization steps completely. And it contains an internal energy term that is penalized by the deviation from the sign distance equation to the level set equation, and also includes an external energy term that causes the zero level set to move the target image. The result of the evolution of the level set equation is a gradient flow, which minimizes all the energy equations. In this section, the variational level set equation has three advantages over the traditional level set equation. First, the time step can be used to solve the partial differential equation, which accelerates the evolution of the curve [6]. Then, the level set equation can be initialized by a general equation, which is more efficient and practical than the use of the symbolic distance equation. Finally, the evolution of the level set in our equation can be performed by the finite difference method, and the computation is more efficient.

In image segmentation, active contour is a dynamic curve moving to the target boundary. In order to achieve this goal, the external energy is defined accurately, which can make the zero level curve moves to the target boundary of the image [7]. Make $I$ as an image, and $g$ is defined as the marginal exponential equation

$$
g=\frac{1}{1+\left|\nabla G_{\sigma} * I\right|^{2}}
$$

where $G_{\sigma}$ is the Gauss kernel of standard deviation $\sigma$. The external energy $\phi(x, y)$ of equation is defined

$$
\varepsilon_{g, \lambda, v}(\phi)=\lambda L_{g}(\phi)+v A_{g}(\phi)
$$

where $\lambda>0$ and $v$ is constant, while $L_{g}(\phi)$ and $A_{g}(\phi)$ are defined

$$
L_{g}(\phi)=\int_{\Omega} g \delta(\phi)|\nabla \phi| d x d y
$$

and

$$
A_{g}(\phi)=\int_{\Omega} g H(-\phi) d x d y
$$

In contrast, where $\delta$ is a unary pulse function, $\mathrm{H}$ is the Hewice function. Total energy equation is

$$
\varepsilon(\phi)=\mu P(\phi)+\varepsilon_{g, \lambda, v}(\phi)
$$

The external energy $\varepsilon_{g, \lambda, v}$ causes the zero level set to move towards the target boundary of the image [8], while the internal energy term $\mu P(\phi)$ is used to compensate the deviation of the function $\phi$ and the symbolic distance equation in evolution.

In order to make the geometric meaning of $L_{g}(\phi)$ easier to understand, we assume that the zero level set of $\phi$ can be represented by a differentiable parameter curve $C(P)$, where $P \in[0,1]$. It is not difficult to know that the energy equation $L_{g}(\phi)$ can calculate the length of the zero level set of $\phi$ function, and the energy equation $A_{g}(\phi)$ in Eq. (6) is used to accelerate the curve evolution. It should be noted that when the equation $g$ is constant 1, the energy function in Eq. (6) is used in the region of $\Omega_{\phi}^{-}=\{(x, y) \mid \phi(x, y)<0\}$ [9], and the energy functional $A_{g}(\phi)$ in Eq. (6) can be considered as the weighted area of $\Omega_{\phi}^{-}$. The coefficient $v$ of the energy functional $A_{g}$ can be positive or negative, depending on the relative distance between the initial curve and the target. If the initial curve is outside the target, the coefficient $v$ should be taken as a positive value in the weighted area, so that the contour can be reduced quickly. And if the initial curve is within the target, the coefficient $v$ 
should be negative to accelerate the contour expansion. By means of the variational method, the derivative of the function $\varepsilon$ can be written as

$$
\frac{\partial \varepsilon}{\partial \phi}=-\mu\left[\Delta \phi-\operatorname{div}\left(\frac{\nabla \phi}{|\nabla \phi|}\right)\right]-\lambda \delta(\phi) \operatorname{div}\left(g \frac{\nabla \phi}{|\nabla \phi|}\right)-v g \delta(\phi)
$$

where $\Delta$ is Laplace operator, thus minimizing the equation $\phi$ satisfies the Euler Lagrange equation $\partial \varepsilon / \partial \phi=0$. The gradient current, which is the steepest descent procedure for minimizing the function $\varepsilon$, is [10]

$$
\frac{\partial \phi}{\partial t}=\mu\left[\Delta \phi-\operatorname{div}\left(\frac{\nabla \phi}{|\nabla \phi|}\right)\right]-\lambda \delta(\phi) \operatorname{div}\left(g \frac{\nabla \phi}{|\nabla \phi|}\right)-v g \delta(\phi)
$$

This is an evolution equation of the level set in this method. The second and third items on the left of Eq. (8) are equivalent to the gradient flow corresponding to the energy functional $\lambda L_{g}(\phi)$ and $v A_{g}(\phi)$, which is mainly used to move the zero level curve to the target boundary. The first term in Eq. (9) is related to the internal energy $\mu P(\phi)$, and the gradient flow is

$$
\Delta \phi-\operatorname{div}(\nabla \phi /|\nabla \phi|)=\operatorname{div}[(1-1 /|\nabla \phi|) \nabla \phi]
$$

where $(1-1 /|\nabla \phi|)$ is diffusivity. If $|\nabla \phi|>1$, then the diffusion rate is positive and its influence is the conventional diffusion, that is, it makes the function $\phi$ to be smoother and then makes the gradient $|\nabla \phi|$ to decrease. If $|\nabla \phi|<1$, it will be the reverse diffusion and then increase the gradient.

\section{Experimental Results}

The variational level set method has been applied to the segmentation of various real bacteria in different forms, and their experimental results are given (see Fig. 2).

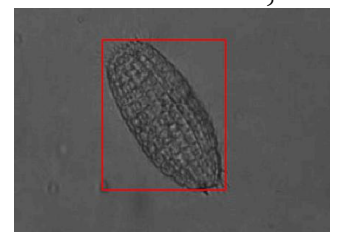

Colepidae

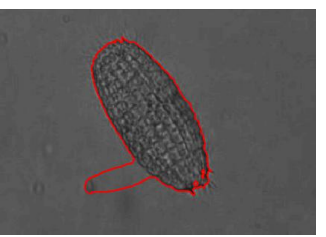

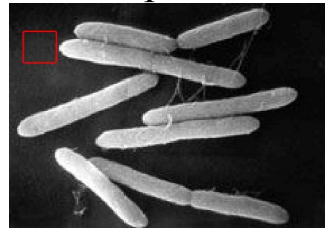

Bacillus coli

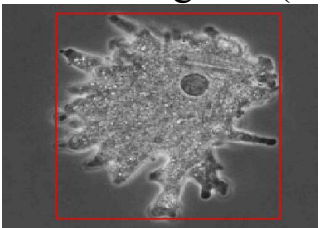

Amoeba

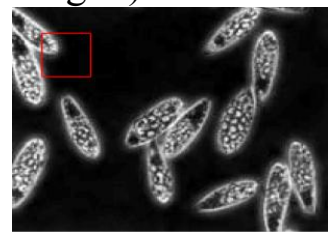

Paramecium

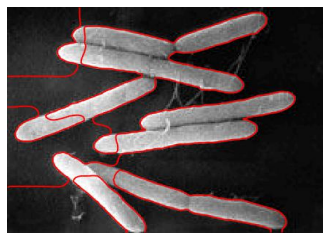

(a) Original drawing
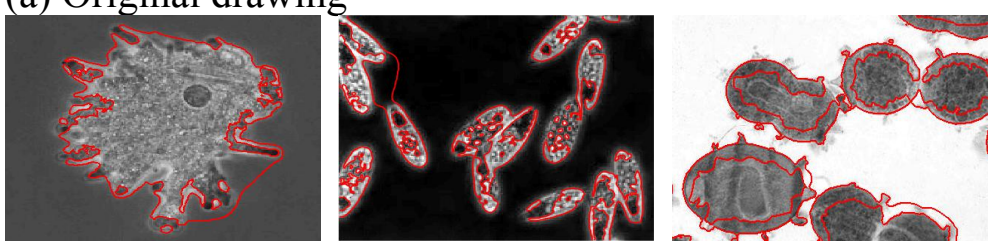

(b) Segmentation results of LBF variational level set

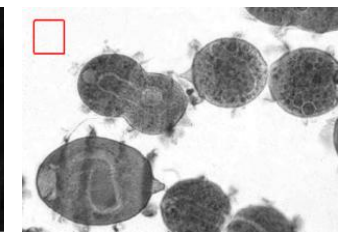

Didinium

Fig. 2 Various bacterial segmentation results of LBF variational level set.

It can be seen that partial boundary of the two cell in some images are somewhat blurred, and the proposed method is robust to the weak boundary image segmentation. In the fourth column, the straight line of the initial level set successfully evolves to the target boundary, and its shape is also repaired very well. The results show that this method has a good performance in extracting the weak boundary of the image, which is not achieved by traditional methods.

\section{Conclusions}

A variational level set equation, which can completely eliminate the re initialization step, is presented in this paper. Compared with the traditional level set equation, it can be separated by a simple finite difference method and is computationally efficient. A large time step is used to accelerate the evolution of the curve while maintaining stability and the level set equation does not need to be initialized as a signed distance equation. This not only makes the calculation more efficient than the symbolic distance equation, but also makes it more flexible. Compared with $\mathrm{C}-\mathrm{V}$ model, the proposed 
model is more robust and more efficient in the computation of bacterial images, especially in the weak boundary and strong noise images.

\section{References}

[1] B. B. Kimia, A. Tannenbaum, and S. Zucker: Shapes, shocks, and deformations I: the components of twodimensional shape and the reaction-diffusion space. International Journal of Computer Vision, vol. 15(1995), p.189-224

[2] C. Li, C. Xu, C. Gui, and M. D. Fox: Level set evolution without re-initialization: A new variational formulation. In IEEE Computer Society Conference on Computer Vision and Pattern Recognition, vol. 1(2005), p. 430-436

[3] V. Caselles, R. Kimmel, and G. Sapiro: On geodesic active contours. International Journal of Computer Vision, vol. 22(1997), p. 61-79

[4] M. Kass, A. Witkin, and D. Terzopoulos: Snakes: Active contour models. International Journal of Computer Vision, vol. 1(1988), p. 321-331

[5] S. Osher and J. A. Sethian: Fronts propagating with curvature-dependent speed: Algorithms based on Hamilton-Jacobi Formulation. Journal of Computational Physics, vol. 79(1988), p. $12-49$

[6] C. Li, C. Kao, J. C. Gore, and Z. Ding: Implicit active contours driven by local binary fitting energy. In IEEE Conference on Computer Vision and Pattern Recognition, no. 1(2007), p. 1-7

[7] V. Caselles, R. Kimmel, and G. Sapiro: On geodesic active contours. nternational Journal of Computer Vision, vol. 22(1997), p. 61-79

[8] H.-K. Zhao, T. Chan, B. Merriman, and S. Osher: A variational level set approach to multiphase motion. Journal of Computational Physics, vol. 127(1996), p. 179-195

[9] G. Aubert and L. Vese: A variational method in image recovery. Siam Journal on Numerical Analysis, vol. 34, no. 5, pp. 1948-1979. SIAM Publisher, Philadelphia(1997)

[10] M. Sussman, P. Smereka, and S. Osher: A level set approach for computing solutions to incompressible two-phase flow. Journal of Computational Physics, vol. 119(1994), p. 146-159 